\title{
Sensitivity Analysis using Sobol indices for the thermal modelling of an Electrical Machine for sizing by optimization Leguyadec $^{(1,2)}$, M. , Gerbaud. L. ${ }^{(2)}$, Vinot. E. ${ }^{(1)}$, Delinchant, B. ${ }^{(2)}$
}

(1) Eco-7,IFSTTAR, Bron,France

(2) Grenoble University, Grenoble, France

\begin{abstract}
Purpose - The thermal modelling of an electrical machine is difficult because the thermal behavior depends on its geometry, the used materials and its manufacturing process. In the paper, such a thermal model is used during the sizing process by optimization of a Hybrid Electric Vehicle (HEV). The paper deals with the sensitivities of thermal parameters on temperatures inside the electrical machine to allow the assessment of the influence of thermal parameters that are hard to assess.

Design/methodology/approach - A sensitivity analysis by Sobol indices is used to assess the sensitivities of the thermal parameters on electrical machine temperatures. As the optimization process needs fast computations, a lumped parameter thermal network (LPTN) is proposed for the thermal modelling of the machine, because of its fastness. This is also useful for the Sobol method that needs too many calls to this thermal model. This model is also used in a global model of a hybrid vehicle.
\end{abstract}

Finding - The difficulty is the thermal modelling of the machine on the validity domain of the sizing problem. The Sobol indices allow to find where a modelling effort has to be carried out.

Research limitations/implications - The Sobol indices have a significant value according to the number of calls of the model and their type (first order, total, etc.). Therefore, the quality of the thermal sensitivity analysis is a compromise between computation times and modelling accuracy.

Practical implications- Thermal modelling of electrical machine in a sizing process by optimization

Originality/value - The use of Sobol indices for the sensitivity analysis of the thermal parameters of an electrical machine.

Keywords: electrical machine, thermal modelling, LPTN, Sobol indices

Paper type application paper

\section{Introduction}

Sizing a hybrid vehicle and its components has proved to be a complex process. To be efficient, this requires considering the system level, the management of the system, and the component level (Silvas et al., 2014, Akli et al., 2009). Moreover, multiphysics aspects have to be taken into account, such as the mechanical model of the vehicle or the engine, the electromagnetic model of the electrical machine (EM) and the thermal aspects of the components. To guarantee a good design, all these aspects are often considered in the same optimization process (Silvas et al., 2014, Song et al., 2018). The drawbacks are the complexity of this approach and the associated computer effort. Because of this, simplified models of the components have to be developed to be inserted in a global optimization process.

In this scope, in (Reinbold et al., 2016, Vinot, 2016), a global optimization method has been developed. It includes a cybernetics approach to represent the vehicle. The problem of energy management (electrical mode choice and battery power choice) is embedded as a sublevel of an optimization to assess the optimal fuel consumption of a given vehicle architecture. A sizing parameterized electromagnetic model of the electrical machine is used to calculate the losses and the maximum performances. A lumped thermal model (Mellor, 1991, Staton et al; 2005) is linked to this electromagnetic model to assess the maximum available torque of the EM in steady state operating. Whereas the validation of the electromagnetic model is relatively easy by means of measurements and finite element method simulation, it is more difficult to develop accurate thermal models due to the uncertainty of many parameters. The accuracy of these models and their sensitivities to different parameters have then to be assessed. However, simple analytical models are sometimes difficult to define, mainly in the science of heat where some parameters may be empiric. So, the sensitivity analysis is useful to decide of the modelling effort (so a computation effort) and the parameters to be considered in the optimization process (which impacts the optimization problem size (Delinchant, 2014)). This paper focuses on the thermal model of the electrical machine and its validation, and the use of the Sobol method (Sobol, 1993) to study the parameter sensitivities for this model (Delinchant et al, 2014).

The second part presents the principle of the Sobol sensitivity analysis approach. The third part describes the modelling and optimization levels referring to previous works. Then, it focuses on the thermal modelling. Finally, the fourth part presents a sensitivity analysis of the thermal model.

\section{Sensitivity analysis using Sobol indices}

\section{II.1. Sensitivity analysis general framework}

A simulation model can be represented by a function:

$$
y=f(\mathbf{x})
$$


Where $y$ is one output of the model to analyze, and $x=\left(x_{1}, x_{2}, \ldots, x_{n}\right)$ is a vector whose size equals $n$. Each $\mathrm{x}_{\mathrm{i}}$ is a random variable defined by a distribution law (e.g.: Gaussian, Uniform, etc.).

The uncertainty analysis tries to give knowledge on the result obtained by a model, whereas the sensitivity analysis tries to give what are the main sources of uncertainty of the selected parameters. So, a sensitivity analysis can be useful to extract the more influential parameters among the $\mathrm{x}$ components that modify the model outputs $(\mathrm{Y})$.

\section{II.2. Sobol indices definition}

There are different ways to measure the importance of an input variable on the outputs. To do that, one needs an indicator that can be qualitative (eg: Morris plan) or quantitative (eg: Sobol indices). The paper focuses on the Sobol indice method (Sobol, 1993, Sobol, 2001). These indices are global because they are not based on any hypothesis on the distribution of parameters (apart from their independence). These indices are quantitative because they are interpreted as the part of the variance due to an input or a group of factors of inputs.

$E\left(y \mid x_{i}\right)$ is the conditional expectation. It is the function depending only on the input $\mathrm{x}_{\mathrm{i}}$, which best approaches the output $y$. The error of prediction is then characterized by the expectation of its square:

$$
E\left[\left(y-E\left(y \mid x_{i}\right)\right)^{2}\right]
$$

The simple rules of calculation on the conditional expectations give:

$$
E\left[\left(y-E\left(y \mid x_{i}\right)\right)^{2}\right]=\operatorname{Var}(y)-\operatorname{Var}\left(E\left(y \mid x_{i}\right)\right)
$$

So, the bigger $\operatorname{Var}\left(E\left(y \mid x_{i}\right)\right)$ is, the better the prediction is. Therefore, $\operatorname{Var}\left(E\left(y \mid x_{i}\right)\right)$ would be the variance of the output $\mathrm{y}$ if it was only a function of $\mathrm{x}_{\mathrm{i}}$. The closer this quantity is to $\operatorname{Var}(y)$, the better the $\mathrm{x}_{\mathrm{i}}$ factor explain the variance of $\mathrm{y}$. In other words, in such a case, the factor $\mathrm{x}_{\mathrm{i}}$ is influential.

The first order Sobol indice is then defined by the ratio of these two quantities:

$$
S_{i}=\frac{\operatorname{Var}\left(E\left(y \mid x_{i}\right)\right)}{\operatorname{Var}(y)}
$$

\section{II.2. Sobol indices calculation}

These indices are based on the interpretation of the conditional expectation in terms of forecast. To calculate the Sobol indices, the variance of the outputs of the model is used. This calculation needs the independence of the inputs, but has no constraints on their distribution. Here, the indices are estimated using sampling such as Quasi Monte Carlo (Grandjacques, 2014). The estimators of expectation and variances require a high number of samples. So, the used model has to be fast. If the model is a numerical one or requires high computation time, it is recommended to use meta-modelling such as Polynomial Chaos (Sudret, 2018) or a frequency technic named RBD-FAST (Random Balance Designs - Fourier Amplitude Sensitivity Test) (Mara, 2009). In our case, the Peek and Freeze technic has been used directly on our fast model to approximate the conditional expectation (Gamboa et al, 2016). This technic is based on the following equality.

$$
\begin{aligned}
\operatorname{Var}\left(E\left(y \mid x_{i}\right)\right) & =E\left(E\left(y \mid x_{i}\right)^{2}\right)-E\left(E\left(y \mid x_{i}\right)\right)^{2} \\
& =E\left(E\left(y \mid x_{i}\right)^{2}\right)-E(y)^{2} \\
& =E\left(Y_{1} Y_{2}\right)-E\left(Y_{1}\right) E\left(Y_{2}\right)=\operatorname{Cov}\left(Y_{1}, Y_{2}\right)
\end{aligned}
$$

Where $\mathrm{Y}_{1}$ respectively $\mathrm{Y}_{2}$ are obtained with the model simulation from random samples $\mathrm{X}_{1}$, resp. $\mathrm{X}_{2}$ of $\mathbf{x}$ in which the values of $\mathrm{x}_{\mathrm{i}}$ (the parameter of interest) are kept the same for both $\mathrm{X}_{1}$ and $\mathrm{X}_{2}$.

$$
S_{i}=\frac{\operatorname{Cov}\left(Y_{1}, Y_{2}\right)}{\operatorname{Var}\left(Y_{1}\right)}
$$

The Pick and Freeze estimator consists in taking an empirical estimator of the numerator and the denominator. This method is robust ([68]) and its speed of convergence does not depend on the number of input variables. The basic estimator of the first order Sobol indice is defined by: 


$$
\hat{S}_{i}=\frac{\frac{1}{N} \sum_{i=1}^{N} Y_{1} Y_{2}-\left(\frac{1}{N} \sum_{i=1}^{N} Y_{1}\right)\left(\frac{1}{N} \sum_{i=1}^{N} Y_{2}\right)}{\frac{1}{N} \sum_{i=1}^{N} Y_{1}^{2}-\left(\frac{1}{N} \sum_{i=1}^{N} Y_{1}\right)^{2}}
$$

The quality of the estimator is based on the used sampler. Sobol advocates the use of samplers with low discrepancies also named quasi-Monte Carlo samplers. In addition, to guarantee the quality of the estimator, it is recommended to use the Bootstrap technique (Efron, 1993) which provides a confidence interval for the estimated magnitudes.

\section{Global optimization and modelling of hybrid vehicle and the electrical machine}

\section{III.1. Global optimization process}

As in many complex systems, Hybrid Electric Vehicle (HEV) design needs to address different levels of modelling (system and components) and optimization (system and control). Moreover, it is usually a multiobjective problem to find the best compromise between fuel consumption, battery size, driving comfort, system cost, component volumes...

Here, a parallel hybrid vehicle architecture is chosen (see Figure 1). System level parameters (power of engine $\mathrm{P}_{\mathrm{ICE}}$, battery size $\mathrm{N}_{\mathrm{BAT}}$, DC voltage $\mathrm{U}_{\mathrm{DC}}$ and gear ratio $\mathrm{k}_{\mathrm{CPL}}$ and $\mathrm{k}_{\mathrm{RED}}$ ) are jointly designed with EM geometrical parameters, using an optimization algorithm $\left(\mathrm{X}_{\mathrm{EM}}\right)$ (Reinbold et al., 2016). The energy management control level which defines the vehicle operating mode (hybrid or all electric) and the battery current (charge or discharge) for each driving condition of a driving cycle have also to be addressed to assess the fuel consumption.

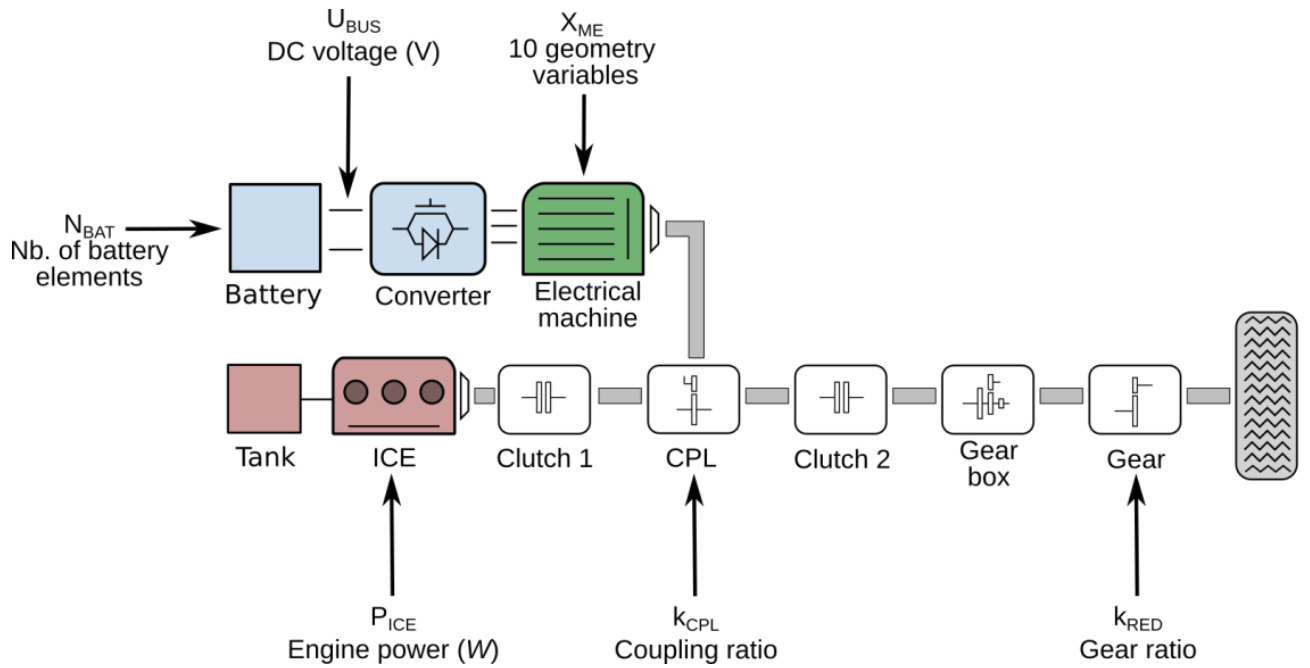

Figure 1: Parallel Hybrid Vehicle power train and chosen sizing parameters

In such a case, a sequential design leads to a suboptimal solution, and a global approach has proved to be more efficient. A good and frequently used approach to solve the bi-level optimization problem consists of an optimization level for the system and component parameters managed by a genetic algorithm and an optimization sublevel for the control part managed by a dynamic programming method (Silvas et al., Vinot, 2016, akli et al., 2009). Figure 2 presents the process developed in previous works and its connection with a thermal model. The genetic algorithm, NSGA-II, is used to design system parameters (gear ratio, engine size...).

There, the model of the electrical machine is a loss map linked to a maximum torque vs. speed characteristic. Using a Reluctance network (RN) model previously developed and validated (Reinbold et al., 2016), the losses are calculated on a sampled torque vs. speed area.

A first maximum torque vs. speed characteristic is then calculated based on a maximum DC voltage limitation with no thermal limits. It is used in the global process (Figure 2) to assess the vehicle performance constraints (maximum acceleration, "perfo. model" in Figure 2 using the direct system model).

A thermal model is used to generate a steady state maximum torque characteristic with a thermal limit ("steady state model" in figure 2) used in the fuel consumption assessment. For that, dynamic programming (Bellman, 1957, Vinot, 2015) is used in order to compute the minimum fuel consumption of the vehicle in a known in advance driving cycle. As this operating cycle is representative of steady state driving conditions, the thermal constraint in the EM has to be respected in a steady state fashion. 
A key point of such an approach is the accuracy of the model and the required computer efforts. To tackle this issue, a parameterized thermal resistance network validated with finite element method and/or measurement, offers a good solution (sections III.2 to III.4).

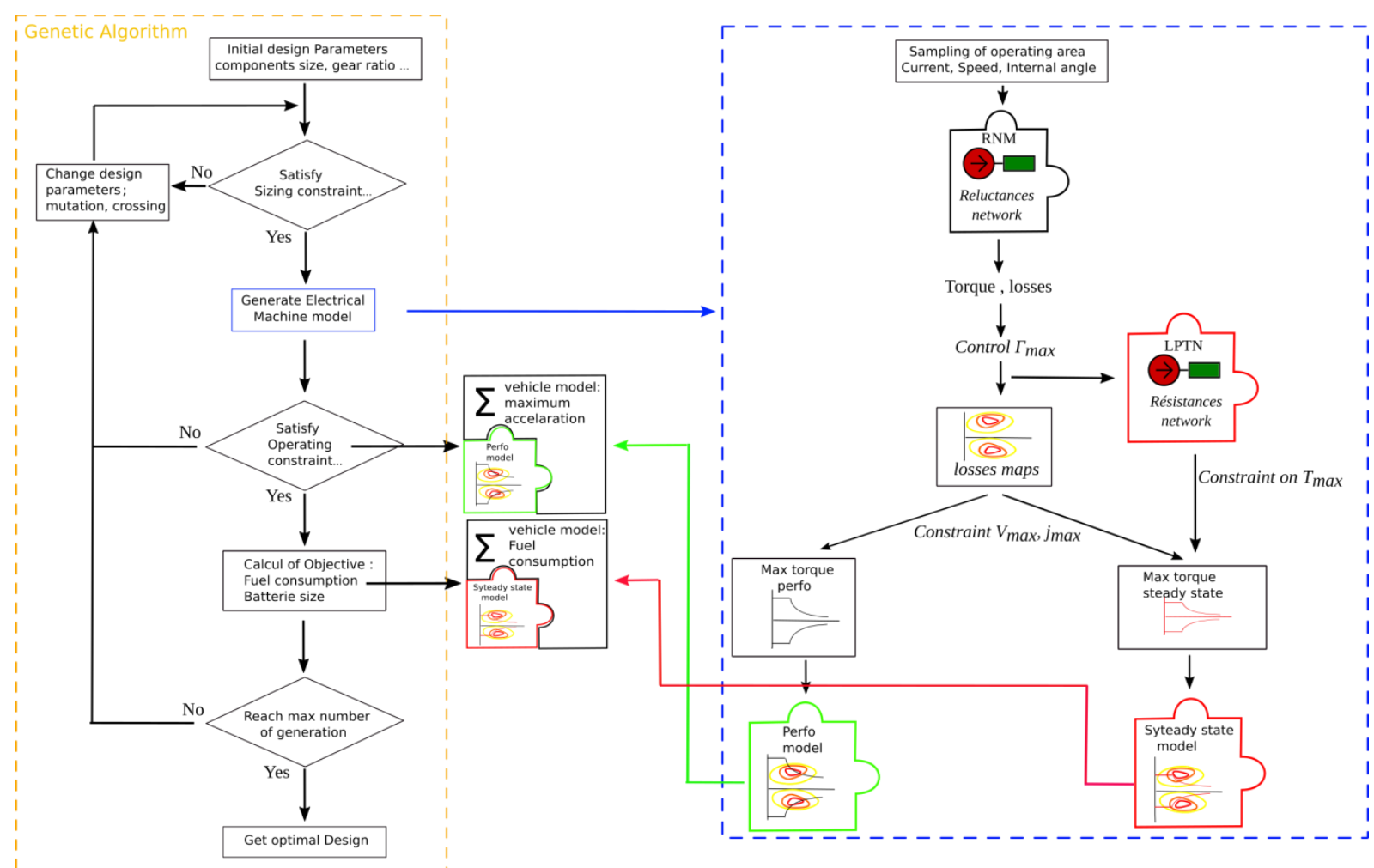

Figure 2: Global optimization process

\section{III.2. Principle of LPTN modelling}

To represent the thermal exchanges and to assess the maximum temperature in the steady state condition, a fast analytical thermal model, based on a lumped parameter thermal network (LPTN) is used (Mellor et al., 1991, Staton et al., 2005, Boglietti et al., 2009, Nategh et al., 2012). The LPTN is an analogy between thermal and electrical phenomena. For instance, temperature is equivalent to voltage and heat flow is equivalent to current. Thermal resistances depend on the considered thermal mode and the sizes of the exchange areas. The following equations are used to calculate the thermal resistances of the model, where $\lambda$ is the thermal conductivity coefficient, and $h$ is the thermal convection coefficient:

- $\quad$ conduction mode through a parallelepiped of length $\mathrm{E}$ and with a section $\mathrm{S}$ :

$$
R_{c d, p l p}=\frac{E}{\lambda \times S}
$$

- conduction mode through a cylinder (radial), with an external radius $\mathrm{R}_{\mathrm{ext}}$, an internal radius $\mathrm{R}_{\mathrm{int}}$ and an angle $\alpha$ :

$$
R_{c d, c y l}=\frac{\ln \left(\frac{R_{\text {ext }}}{R_{\text {int }}}\right)}{\alpha \times \lambda \times S}
$$

- $\quad$ convection mode on a surface $S$ :

$$
R_{c v}=\frac{1}{h \times S}
$$

An analytical calculation is performed to find the temperature at every node of the equivalent circuit. In fact, it consists of solving the system of linear equations deduced from the Kirchoff's law applied at every circuit node. 


\section{III.3. Application of the LPTN modelling}

The LPTN model has been applied to a permanent magnet synchronous machine commonly used in HEV. The machine used in this example is a $50 \mathrm{~kW}$ machine $(85 \mathrm{~mm}$ depth, diameter of $272 \mathrm{~mm}$, see more details in Reinbold et al., 2016). Figure 3 shows a representation of the axial section of the EM and of the radial section of one slot. The sizes are not strictly respected in this figure which aims to show all the EM parts and the temperature points. The casing is cooled thanks to water channels whose temperature is externally controlled. The copper losses (CL) and the iron losses (IL) generate heat into the machine. The heat paths are represented by equivalent thermal resistances:

- $\quad$ conduction in dark blue

- contact in light blue

- convection in green.

Oblique lines represent orthoradial heat exchanges in the stator (between tooth and slot as an example). The internal cooling is ensured by an oil/air mixture (oil fog) which is splattered on the end-windings (yellow).
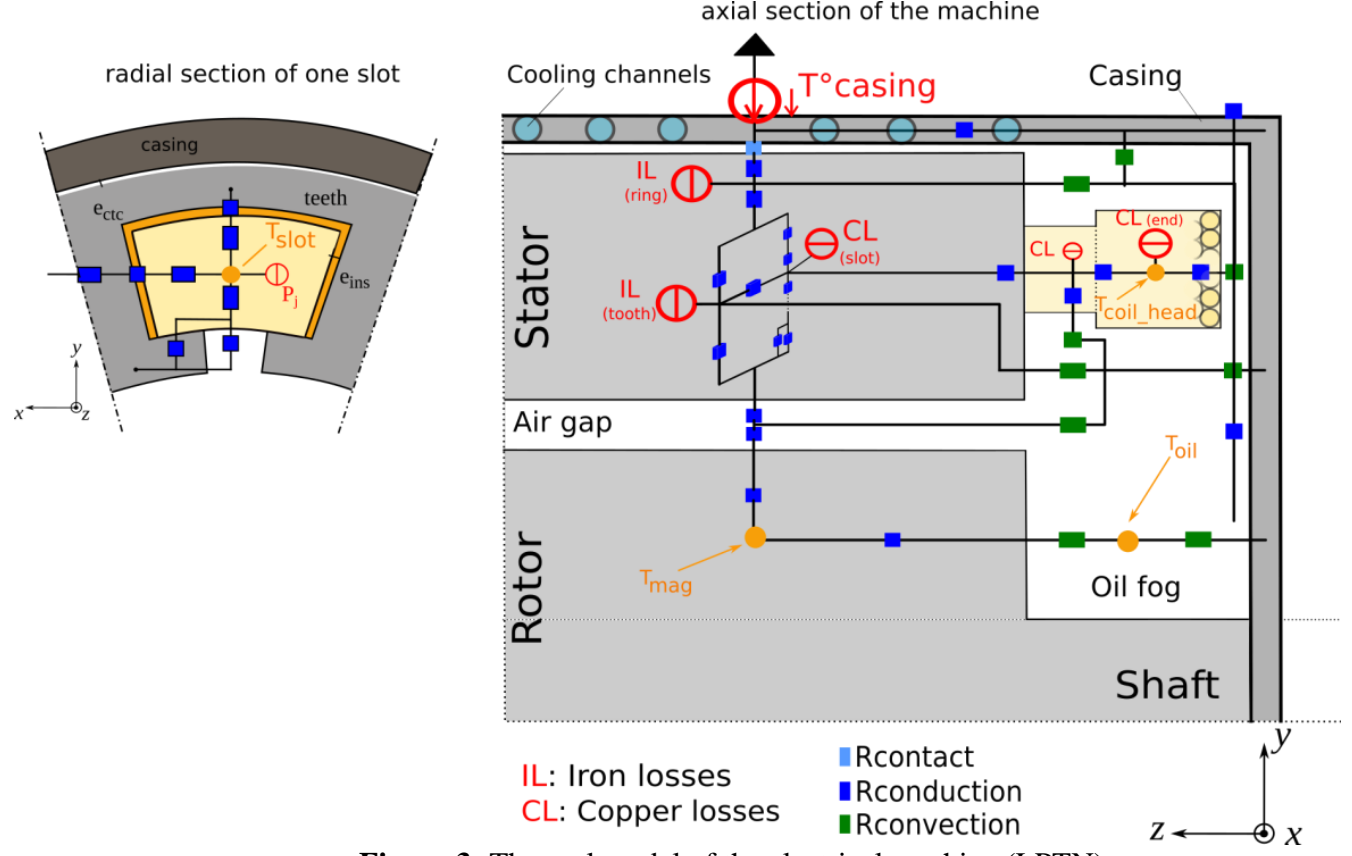

Figure 3: Thermal model of the electrical machine (LPTN)

The LPTN model gives the temperature of several areas in the machine. The critical areas (i.e. where the temperature has to be under a threshold) are:

- the windings $\left(\mathrm{T}_{\text {coil_head }}<170^{\circ} \mathrm{C}\right.$ and $\mathrm{T}_{\text {slot }}<170^{\circ} \mathrm{C}$ ),

- the inner oil $\left(\mathrm{T}_{\text {oil }}<158{ }^{\circ} \mathrm{C}\right)$

- the permanent magnets $\left(\mathrm{T}_{\mathrm{mag}}<220^{\circ} \mathrm{C}\right)$.

The model inputs are geometrical variables and operating losses given by the electromagnetic model (see part II). Previously to its use with the global process, this model was validated by means of finite element model (FEM) (simulation).

\section{III.4. Validation of LPTN modelling}

A finite elements model has previously been developed (Le-Guyadec et al., 2017). It has been useful to understand the main path of the thermal exchanges and then to adjust the topology of the LPTN model (coil-head and slot modeling). A comparison of the temperatures and the thermal exchanges in different areas of the machine has been performed for different operating points and different geometries of the EM (see Figure 4).

For the LPTN, the temperatures are those of the nodes, whereas for the finite element model (FEM) they are the average temperatures of the areas. Here, the sizes of the machine are from a reference machine described in (Hsu et al., 2005) with $935 \mathrm{~W}$ of Joule losses (assumed uniform in the winding) and $84 \mathrm{~W}$ of iron losses. The rotor iron losses are negligible in a permanent magnet machine thus the iron losses are uniformly assigned in the stator.

The differences between the temperatures computed with the two models are less than $5 \%$. The thermal paths are also globally in good agreement with the LPTN model compared to the FEM. Figure 6 shows the power exchanged between different areas of the machine described in Figure 5 (the number in the x-axis Figure 6 corresponds to the thermal path numbered in Figure 5). The main difference is observed for the exchange between the slots and the stator (path 1): a difference of $15 \%$ which remains acceptable. The same comparison has been 
performed for different operating points (i.e. involving mainly iron losses) and different geometries of the machine, mainly concerning the ratio length/diameter to simulate different machine shapes as in an optimization process. The differences between the model remain lower than $15 \%$ and the main disparities appear for "extreme" geometries not reachable in the design space (EM with high length compared to diameter).

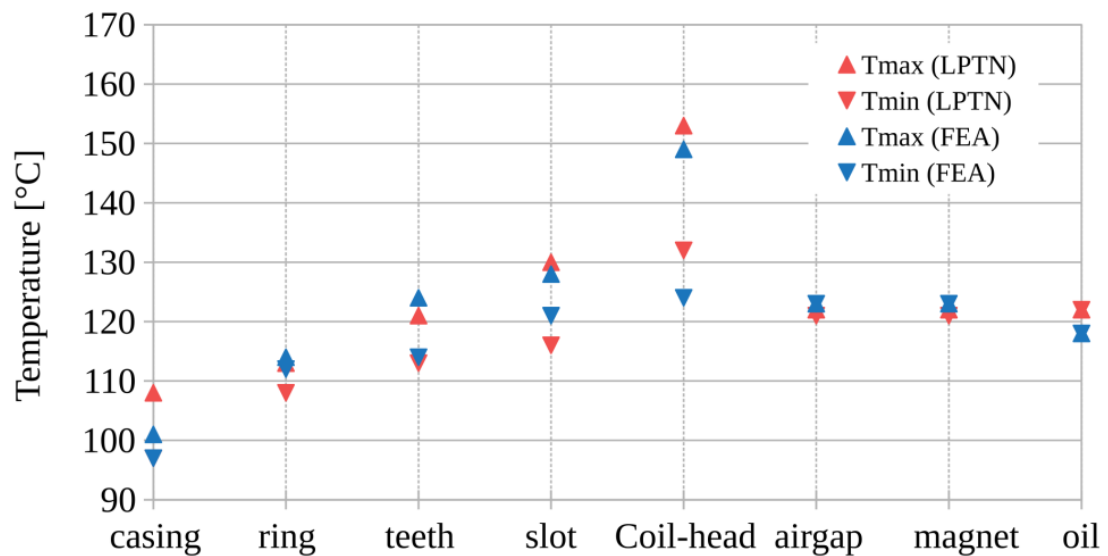

Figure 4: Temperatures in different area of the machine obtained by FE model and LPTN model

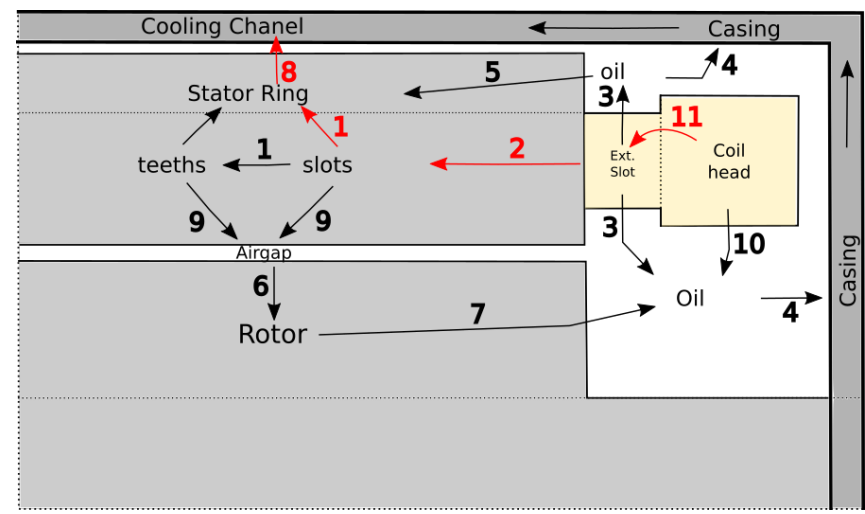

Figure 5: Thermal paths in the electrical machine

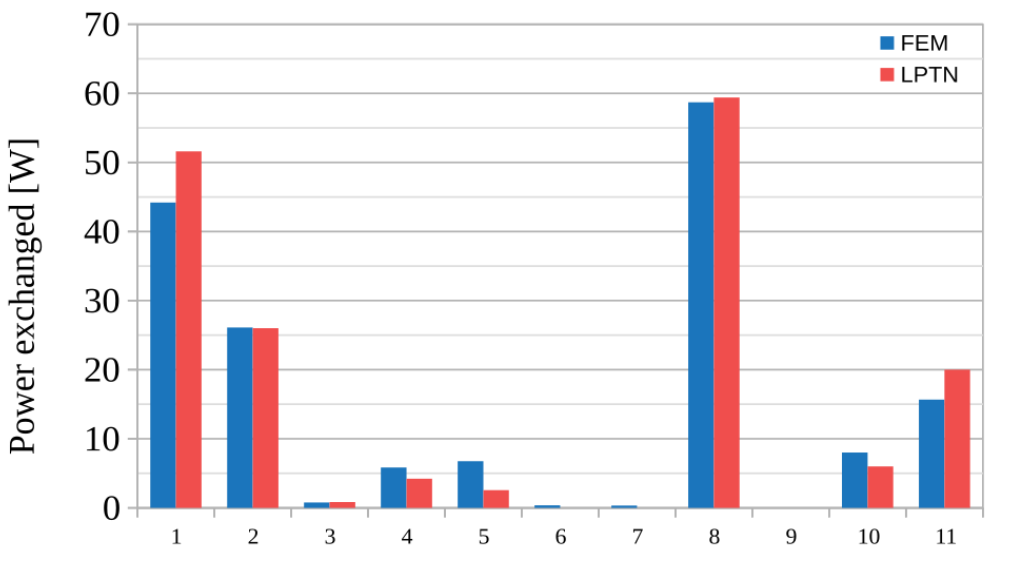

1: slot $->$ Stator

2: ext. slot $->$ slot

3: ext slot $->$ oil

4: oil -> casing

5: oil -> stator

6: rotor $->$ airgap

7: rotor $->$ oil

8: stator $->$ casin

9:stator -> airgap

10: coil head $->$ oil

11:coil head -> ext. slot

Figure 6: Thermal paths comparison between LPTN and FEM

Even with the previous validations, some input parameters of the model (thermal exchange coefficients or thickness of certain areas) remain difficult to assess with accuracy. In order to know which parameters are critical to assess or which parameters have little effect on the temperatures, a sensitivity analysis was performed (see part IV).

\section{SENTITIVITY ANALYSIS}

\section{IV.1. Uncertainty analysis}

An a priori study allowed the extraction of four influential parameters:

- $h_{\text {oil }}$, thermal convection coefficient between the end windings and the inner oil, 
- $\quad \mathrm{e}_{\mathrm{ctc}}$, thickness of the contact between the stator and the casing,

- $\quad \mathrm{e}_{\mathrm{ins}}$, thickness of the insulation between slots and teeth,

- $\quad \lambda_{\text {radcd }}$, equivalent thermal conduction of the windings in the plane normal to the winding axis.

The ranges of variation of these parameters are presented in Table 1 and correspond to typical values found in literature.

Table 1: Range parameters variations

\begin{tabular}{|c|c|c|}
\hline Parameters & Units & range \\
\hline $\mathrm{h}_{\text {oil }}$ & $\mathrm{W} \cdot \mathrm{m}^{-2} \cdot{ }^{\circ} \mathrm{C}^{-1}$ & {$[25 ; 100]$} \\
\hline $\mathrm{e}_{\mathrm{ctc}}$ & $\mathrm{W} \cdot \mathrm{m}^{-1} \cdot{ }^{\circ} \mathrm{C}^{-1}$ & {$[1.6 ; 32]$} \\
\hline $\mathrm{e}_{\text {ins }}$ & $\mathrm{m}$ & {$\left[6.10^{-5} ; 12.10^{-5}\right]$} \\
\hline$\lambda_{\text {radcd }}$ & $\mathrm{m}$ & {$\left[19.10^{-5} ; 50.10^{-5}\right]$} \\
\hline
\end{tabular}

The samples are generated by the semi Monte-Carlo method as explained in Section II.2. In our application, the sensitivity analysis is carried out for four parameters, i.e. $\mathrm{p}=4$. $\mathrm{N}=10^{6}$ samples are generated. For that, two $\mathrm{p}$ by $\mathrm{N}$ matrixes $\left(\mathrm{X}_{1}\right.$ and $\mathrm{X}^{2}$ ) are generated in a completely random manner corresponding to $\mathrm{N}$ values of quadruplets $\left[\mathrm{h}_{\mathrm{oil}}, \mathrm{e}_{\mathrm{ctc}}, \mathrm{e}_{\mathrm{ins}}, \lambda_{\text {padcd }}\right]$. Then, to assess the Sobol indices corresponding for example to $\mathrm{h}_{\mathrm{oil}}$, the first column of $\mathrm{X}_{2}$ is replaced by those of $\mathrm{X}_{1}$. The values of the temperature are then assessed using the LPTN model for its two sets of values providing Y1 and Y2 matrix used to calculate the Sobol indices with the method presented section II.2.

As the computation times of a LPTN model are really fast, such a process takes $285 \mathrm{~s}$ to assess the indices corresponding to one parameter $(2 * \mathrm{~N}$ evaluations of the model).

For this distribution of samples, Figure 7 and Table 2 present the average value and the deviation of the temperatures for the four temperature points of the machine (orange points Figure 3) which are the most critical (see section III.3). The two temperatures which have the highest range of variation are the Coil-head and the Slot temperatures corresponding to the windings. They also present the higher deviation.
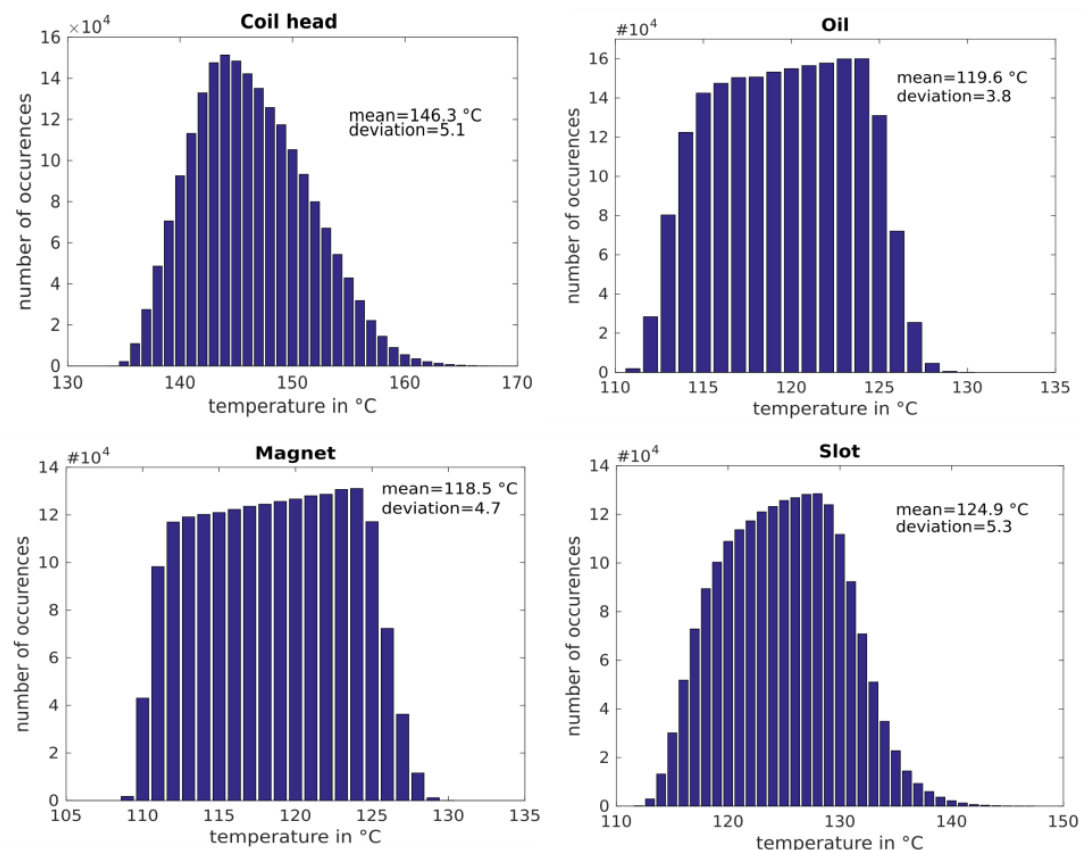

Figure 7: Distribution of the temperatures in coil-head, oil, magnet and slot considering.

Table 2: Results of the uncertainty analysis on the temperatures $\left({ }^{\circ} \mathrm{C}\right)$ inside the electrical machine

\begin{tabular}{|c|c|c|c|c|}
\hline & $\mathrm{T}_{\text {end }}$ & $\mathrm{T}_{\mathrm{mag}}$ & $\mathrm{T}_{\text {oil }}$ & $\mathrm{T}_{\text {slot }}$ \\
\hline Average value & 146.3 & 122.2 & 122.5 & 128.6 \\
\hline Deviation & 5.1 & 2.6 & 2.2 & 3.6 \\
\hline Maximal variation & 13 & 6.4 & 5.4 & 8.4 \\
\hline
\end{tabular}

\section{IV.2. Sensitivity analysis}


A sensitivity analysis allows the identification of the thermal parameters that have the highest influence on the model outputs. So, these parameters have to be accurately estimated to obtain the temperatures of the critical areas of the machine with an acceptable accuracy (in the context of pre-sizing). The obtained results are presented in Table 3 and Figure 8 for three temperatures.

Table 3: First order Sobol indices.

\begin{tabular}{|c|c|c|c|c|}
\hline & $\mathrm{h}_{\text {oil }}$ & $\mathrm{e}_{\text {ctc }}$ & $\mathrm{e}_{\text {ins }}$ & $\lambda_{\text {radcd }}$ \\
\hline $\mathrm{T}_{\text {coil_head }}$ & 0,64 & 0,24 & 0,05 & 0,13 \\
\hline $\mathrm{T}_{\text {oil }}$ & 0,12 & 0,8 & 0,05 & 0,01 \\
\hline $\mathrm{T}_{\text {mag }}$ & 0,18 & 0,89 & 0,1 & 0,11 \\
\hline $\mathrm{T}_{\text {slot }}$ & 0.13 & 0.44 & 0.12 & 0.27 \\
\hline
\end{tabular}

$\mathrm{e}_{\mathrm{ctc}}$ has a great influence on the temperatures of the magnets and the oil. Nevertheless, $\mathrm{h}_{\text {oil }}$ has the strongest influence on the coil-head temperature, which is also the maximal temperature in the machine. On the other hand, $\lambda_{\text {radcd }}$ and $e_{\text {ins }}$ have less influence. So, these two parameters $\left(e_{c t c}\right.$ and $h_{\text {oil }}$ ) have to be carefully chosen to correctly estimate the temperatures. The coil-head area and the contact between stator and casing have to be represented with good accuracy. The contact area between the stator lamination and the casing has also to be manufactured with good care.

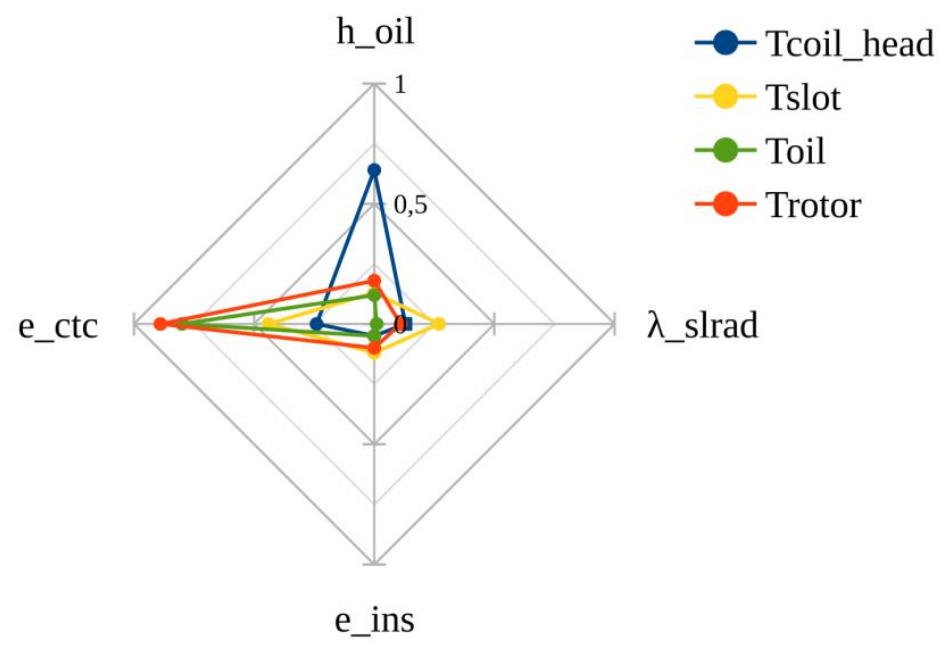

Figure 8: Sobol indices of the LPTN model

\section{Conclusion}

A LPTN model is developed to be implemented in a global optimization process for the sizing of the electrical machine of a hybrid vehicle. First it is validate using Finite Element Method. Then, it is used in the building of the efficiency map of the machine, by suppressing the operating points that cannot be thermally accepted.

As some parameters of this LPTN model can be difficult to assess, a sensitivity analysis using Sobol indices has been performed. After a short presentation of the Sobol method, it is used to assess the influence of four thermal parameters of an electrical machine on critical temperature (windings, magnet, copper). In the paper cases, it shows that the oil thermal convection coefficient has the highest influence on the coil-head temperature which is the most critical in our machines. The temperatures are also sensitive to the contact between stator and casing.

This model could now be used in future work to simulate and design different types of powertrain architectures and their components.

\section{References}

Akli, C. R.; Sareni, B.; Roboam, X. \& Jeunesse, (2009) A. "Integrated optimal design of a hybrid locomotive with multiobjective genetic algorithms", International Journal of Applied Electromagnetics and Mechanics, IOS Press, Vol. 30, pp. 151-162.

Bellman, R. (1657), Dynamic Programming, Princeton University Press, 1957 
Boglietti, A.; Cavagnino, A.; Staton, D.; Shanel, M.; Mueller, M. \& Mejuto, C. (2009) "Evolution and modern approaches for thermal analysis of electrical machines", Transactions on industrial electronics, IEEE, Vol. 56, pp. 871-882

Delinchant, B. (2013) "Reliable Based Design Optimization using k-sigma method and local sensitivity", SAMO'13, 7th International Conference on Sensitivity Analysis of Model Output, July 1-4 2013, Nice

Delinchant, B.; Dogan, H.; Wurtz, F.; Garbuio, L. (2014) "Derivative based global sensitivity analysis for screening and robustness studies of electromagnetic devices”, CEFC'14, 25-28 May, Annecy, France

Efron, B; Tibshirani, R. (1993) “An introduction to bootstrap”. Chapman \& Hall, $1^{\text {st }}$ edition.

Gamboa, F.; Janon, A.; Klein, T.; Lagnoux-Renaudie, A.; Prieur, C. (2016) "Statistical inference for Sobol pick freeze Monte Carlo method, Statistics", Taylor \& Francis: STM, Behavioural Science and Public Health Titles, Vol. $50 \mathrm{~N}^{\circ} 4$, pp.881-902.

Grandjacques, M.; Janon, A.; Adrot, O.; Delinchant, B. (2014) "Pick-freeze estimation of sensitivity indices for models with dependent causal processes inputs", MCQMC 2014 - April 6-11, 2014 - KU Leuven, Belgium

Hsu, J., Ayers C. and Coomer C. (2005), "Report on toyota prius motor design and thermal manufacturing assessment". Federal Register, pp. 1-15.

Le-Guyadec, M.; Vinot, E.; Gerbaud, L.; Lombard, P.; Chaumond, A. \& Boussey, T. (2017) "Building of an electrical machine thermal model in the context of a hybrid electric vehicle global optimization" in Vehicle" Power and Propulsion Conference proceedings of the international conference in Belfort, France, pp. 1-6

Mara, T.A (2009) "Extension of the rbd-fast method to the computation of global sensitivity indices". Reliability Engineering and System Safety, Vol. 94, pp.1274-1281, 2009.

Mellor, P.; Roberts, D. \& Turner, D. (1991), "Lumped parameter thermal model for electrical machines of TEFC design”, IEE Proceedings B (Electric Power Applications), Vol. 138, pp. 205-218

Nategh, S.; Wallmark, O.; Leksell, M. \& Zhao, S. (2012) “Thermal analysis of a PMSRM using partial FEA and lumped parameter modelling” IEEE Transactions on Energy Conversion, Vol. 27, pp. 477-488.

Reinbold, V.; Vinot, V.; Garbuio, L. and Gerbaud, L. (2016), "Optimal sizing of an electrical machine using a magnetic circuit model: application to a hybrid electrical vehicle", IET Electrical Systems in Transportation, Vol. 6, pp. 27-33.

Sobol. M. (1993), "Sensitivity estimates for nonlinear mathematical models", Mathematic Model And Computational Experiment, vol. 1, no4, pp.407-414.

Sobol, M. (2001), "Global sensitivity indices for nonlinear mathematical models and their Monte Carlo estimates", Mathematics and computers in simulation, 2001, vol. 55, no 1-3, p. 271-280.

Silvas, E.; Bergshoeff, E.; Hofman, T. \& Steinbuch, M. (2014) "Comparison of Bi-Level Optimization Frameworks for Sizing and Control of a Hybrid Electric Vehicle" in Vehicle" Power and Propulsion Conference proceedings of the international conference in Coimbra, Portugal, pp. 1-6

Song, Z.; Zhang, X.; Li, J.; Hofmann, H.; Ouyang, M. \& Du, (2018) J. "Component sizing optimization of plugin hybrid electric vehicles with the hybrid energy storage system”, Energy, Vol. 144, pp. 393-403

Staton, D.; Boglietti, A. and Cavagnino, A. (2005) "Solving the more difficult aspects of electric motor thermal analysis in small and medium size industrial induction motors", IEEE Transaction on Energy Conversion, Vol. 20, pp. 620-628

Sudret, B (2018) "Global sensitivity analysis using polynomial chaos expansions". Reliability Engineering and System Safety, Vol. 93, pp. 964-979

Vinot, E. (2015) "Time reduction of the Dynamic Programming computation in the case of hybrid vehicle", International Journal of Applied Electromagnetics and Mechanics, Vol. 53, pp. 1-15

Vinot, E. (2016) "Comparison of different power-split architectures using a global optimization design method", Int. J Electric and Hybrid Vehicles, Vol. 8, pp. 225-241 
